\title{
CORRESPONDENCE
}

\section{Pulmonary lymphangitic carcinomatosis presenting as severe interstitial lung disease in a 15-year-old female}

\section{To the Editor:}

Pulmonary lymphangitic carcinomatosis (PLC) is a metastatic lung disease characterised by the diffuse infiltration and obstruction of the pulmonary parenchymal lymphatic system by tumour cells [1]. It is an extremely rare diagnosis in paediatrics and we believe that this is the first reported case in a child, presenting as interstitial lung disease without a known primary tumour.

A 15-yr-old Caucasian female was transferred to our hospital (Birmingham Children's Hospital, Birmingham, UK) for further assessment and management of respiratory failure, secondary to presumed interstitial lung disease. 8 weeks previously she had presented to her local hospital with a 5week history of cough and shortness of breath associated with lethargy, poor appetite and early satiety. On examination she was hypoxic with bilateral wheeze and crackles. Bilateral perihilar shadows and increased bronchovascular markings were seen on the chest radiograph. Atypical pneumonia was diagnosed and she received oxygen, oral erythromycin and inhaled salbutamol. She recovered but after discharge required two courses of oral prednisolone and salbutamol for wheeze and shortness of breath.

She deteriorated while visiting relatives in the Birmingham area of the UK and required admission for cough, shortness of breath and haemoptysis. On examination she was tachypnoeic, tachycardic and hypoxic but not clubbed. There was generalised decreased air entry and bilateral inspiratory crackles on ausultation. Her blood tests were unremarkable apart from elevated C-reactive protein $\left(54 \mathrm{mg} \cdot \mathrm{L}^{-1}\right)$. A chest radiograph and subsequent computed tomography (CT) scan of her chest showed extensive, bilateral interstitial changes (fig. 1). A diagnosis of presumed interstitial lung disease was made. The differential diagnoses are summarised in table 1.

The patient's family were from the travelling community but lived in a brick house with central heating. Although she was a nonsmoker, her parents smoked heavily. She was not sexually active and denied any solvent or recreational drug use. There had been no recent overseas travel and no infectious contact. Apart from brief contact with pigeons 8 months prior to presentation there was no history of exposure to organic dusts. She admitted poor compliance with thyroxine that had been prescribed after she was diagnosed with hypothyroidism aged 6 yrs.

A wide range of investigations were performed (table 1) including a flexible bronchoscopy and bronchoalveolar lavage. After these procedures the patient required intubation and ventilation with high-frequency oscillation ventilation (HFOV). A blood culture from the referring hospital (Birmingham City
Hospital, Birmingham, UK) grew Haemophilus influenzae and intravenous piperacillin with tazobactam and ciprofloxacin were commenced.

Once bacterial, fungal and Pneuomocystis carinii pneumonia infection were excluded (day 3), high dose i.v methylprednisolone was started. Despite this, the patient continued to deteriorate with worsening respiratory failure. A lung biopsy was discussed at length between members of the paediatric respiratory, intensive care and surgical teams, as well as the family. The consensus was that as the patient was on HFOV and the lesions were peripheral, a transbronchial biopsy would have been technically difficult and unlikely to produce a diagnostic biopsy. It was also thought that the patient's unstable clinical condition meant that an open biopsy carried an unacceptable risk of significant complications or death. Therefore, the biopsy was delayed to allow for any clinical improvement which would decrease these risks. Antimicrobial therapy was extended empirically to cover for possible Mycobacterium tuberculosis, fungal and $P$. carinii infection. By day 14, despite almost maximal HFOV (mean airway pressure $28 \mathrm{cmH}_{2} \mathrm{O}$, amplitude $(\Delta \mathrm{P}) 120$, inspiratory oxygen fraction $100 \%)$, her condition deteriorated further. She required a noradrenaline infusion and chest radiograph showed increased interstitial changes and bilateral pleural effusions. In view of her deteriorating condition and the absence of definitive diagnosis, lung biopsy was reconsidered. It was thought unlikely that the patient's condition would improve and that the patient was at high risk of dying while on the ventilator. After counselling the family about the severe risks, it was decided that an open lung biopsy should be performed in an attempt to make a diagnosis. This was performed on the paediatric intensive care unit on day 16 . She tolerated the procedure without complications.
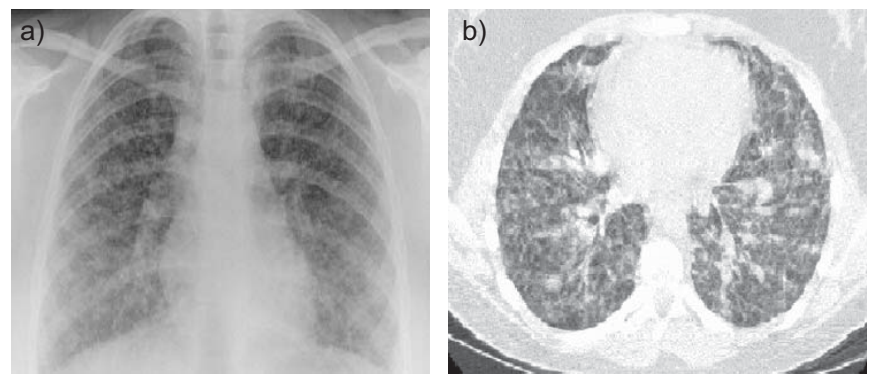

FIGURE 1. a) Chest radiograph showing bilateral coarse interstitial shadowing. b) High-resolution computed tomography scan of the chest. The image is degraded by movement due to patient dyspnoea. The thickened interlobular septae is suggestive of interstitial lung disease. 
TABLE 1 Differential diagnoses, investigations and results

\begin{tabular}{|c|c|c|}
\hline Differential diagnosis & Investigation & Result \\
\hline Bacterial LRTI & BAL microscopy and culture & Negative \\
\hline Fungal LRTI & BAL cytology and culture & Negative \\
\hline PCP & BAL cytology and PCR & Negative \\
\hline Pulmonary TB & BAL acid-fast bacilli stain and culture; Mantoux test & Negative \\
\hline Sarcoidosis & Serum ACE & Normal \\
\hline Alveolar haemorrhage syndrome ${ }^{\#}$ & ANCA, anti-GBM and BAL-haemosiderin laden macrophages & Negative \\
\hline Histiocytosis & Bone marrow aspirate & Normal \\
\hline Immunodeficiency & Igs and HIV tests & Normal \\
\hline Congenital or acquired cardiac abnormality & Echocardiogram & Normal \\
\hline
\end{tabular}

The lung biopsy showed multiple tumour thrombi within the lymphatic vessels, around airways and arteries, and along the pleural surface (fig. 2). A sample of pleural fluid obtained during the procedure revealed large atypical cells, probably epithelial in origin. A diagnosis of PLC from a primary adenocarcinoma was made. The diagnosis and poor prognosis was discussed with the parents. Life support treatment was withdrawn on day 19 and she died shortly afterwards. The family refused a post mortem.

Intrathoracic metastases occur in $30-40 \%$ of patients with malignancy and $6-8 \%$ of these patients have PLC [1]. It most commonly affects patients aged $40-49 \mathrm{yrs}$ and is rare in younger patients. There is one paediatric case report of PLC in a patient with renal adenocarcinoma [2] and a case series of six young adults (average age 26 yrs) with PLC from occult stomach carcinoma [3]. The present case is the first to report PLC in a child from an occult malignancy presenting with symptoms and signs of interstitial lung disease.

The spread of tumour cells to the pulmonary lymphatic system or the adjacent interstitia causes thickening of the bronchovascular bundles and septa. The interstita is further thickened by a
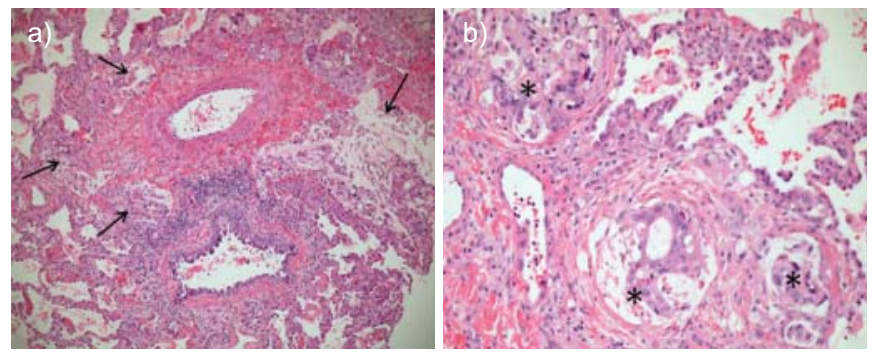

FIGURE 2. Tumour thrombi are seen in lymphatic vessels surrounding a) pulmonary arteries and bronchi (arrows) and b) underneath the pleura $\left(^{*}\right)$. Haematoxylin and eosin stain. desmoplastic reaction caused by the proliferation of tumour cells and lymphatic dilation $[4,5]$. The primary tumours most commonly associated with PLC are: breast (33\%), stomach $(29 \%)$, lungs $(17 \%)$, pancreas $(4 \%)$ and prostate $(3 \%)[1,4]$. The preferred diagnostic procedure for PLC is lung biopsy (transbronchial or open). The pulmonary microvascular cytology may be of value when a lung biopsy is refused or thought to be too hazardous [6].

In this case it was decided that a transbronchial biopsy was unlikely to be diagnostic and an open lung biopsy was therefore favoured. Early in her admission, as it was hoped the patient's condition would improve, an open lung biopsy was thought to carry an unacceptable risk of significant complications or death. This is supported by a recent review which reports the mortality rate of open lung biopsy in mechanically ventilated patients as 54\% [7]. With hindsight it can be argued that despite the patient's condition the biopsy should have been performed on day 3 as she had deteriorated further by the time it was performed on day 16. In practice, lung biopsy should be performed as early as it is practical and safe to do so.

PLC can present with dyspnoea and cough and, as with our patient, this may precede the diagnosis of the primary tumour. In the case series mentioned previously, the most prominent symptom other than progressive dyspnoea was weight loss [3]. Even if histologically confirmed, the chest radiograph is normal in $30-50 \%$ of cases. A variety of chest radiographs and CT changes are reported and can mimic those of sarcoidosis [4]. The prognosis for patients with PLC is extremely poor with less than half surviving past 3 months [1]. Despite this there are case reports of platinum-based chemotherapy leading to transient remission [8].

In summary, PLC is a rare but important cause of respiratory compromise that may present with the signs and symptoms of interstitial lung disease in a child. 
F.J. Gilchrist*, H. Alton", M-A Brundler ${ }^{\natural}$, L. Edwards ${ }^{+}$, A. Plunkett ${ }^{+}$and S. Rao*

Depts of *Paediatric Respiratory Medicine, "Radiology, "Pathology, and 'Paediatric Intensive Care, Birmingham Children's Hospital, Birmingham, UK.

Correspondence: S. Rao, Dept of Paediatric Respiratory Medicine, Birmingham Children's Hospital, Steelhouse Lane, Birmingham, B4 6NH, UK. E-mail: satish.rao@bch.nhs.uk

Statement of Interest: S. Rao has received educational grants from Novartis (UK) and GlaxoSmithKline (UK) for attending conferences and delivering lectures as part of an educational programme organised by the two companies.

Provenance: Submitted article, peer reviewed.

\section{REFERENCES}

1 Bruce DM, Heys SD, Eremin O. Lymphangitis carcinomatosa: a literature review. J $R$ Coll Surg Edinb 1996; 41: 7-13.
2 Vanclaire J, Bodart E, Schlesser P, et al. Lymphangite carcinomateuse pulmonaire et adénocarcinome rénal [Pulmonary carcinomatous lymphangitis and renal adenocarcinoma]. Arch Fr Pediatr 1990; 47: 735-736.

3 Dennstedt FE, Greenberg SD, Kim HS, et al. Pulmonary lymphangitic carcinomatosis from occult stomach carcinoma in young adults: an unusual cause of dyspnea. Chest 1983; 84: 787-788.

4 Thomas A, Lenox R. Pulmonary lymphangitic carcinomatosis as a primary manifestation of colon cancer in a young adult. CMAJ 2008; 179: 338-340.

5 Section VI: Pulmonary neoplasms. In: Fraser RS, Muller NL, Colman NC, et al., eds. Fraser and Pare's Diagnosis of Diseases of the Chest. Philadelphia, Elsevier Health Sciences, 1999; pp. 1390-1397.

6 Masson RG, Krikorian J, Lukl P, et al. Pulmonary microvascular cytology in the diagnosis of lymphangitic carcinomatosis. $N$ Engl J Med 1989; 321: 71-76.

7 Defranchi S, Bertolotti AM, Vigliano CA, et al. Open lung biopsy for diffuse disease in patients with and without previously transplanted solid organs. Ann Thorac Surg 2010; 90: 965-971.

8 Kikuchi N, Shiozawa T, Ishii Y, et al. A patient with pulmonary lymphangitic carcinomatosis successfully treated with TS-1 and cisplatin. Intern Med 2007; 46: 491-494.

\section{Pulmonary nocardiosis in immunocompetent patients: can COPD be the only risk factor?}

\section{To the Editor:}

Pulmonary nocardiosis is a rare disorder that mainly affects immunocompromised patients. Several risk factors have been identified, such as corticosteroid therapy, chronic obstructive pulmonary disease (COPD), cystic fibrosis and bronchiectasis. Diagnosis of nocardiosis is difficult as bacteriological culture can be problematic. However, if observations are atypical, clinicians should consider Nocardia in immunocompetent patients, particularly when the patient is also suffering from COPD. Herein, we ask whether COPD alone is a risk factor for pulmonary nocardiosis.

We report the case of a 71-yr-old female smoker suffering from COPD who had not received inhaled or oral (bolus or long-term) corticosteroid therapy. She had suffered from bronchiectasis after pulmonary tuberculosis 60 yrs previously. She presented to the hospital with an exacerbated chronic cough that had lasted several months and chronic fever, with no weight loss. Prior to admission, amoxicillin followed by spiramycin therapy was not effective. Laboratory evaluation showed hyperleukocytosis $\left(14,130\right.$ cells per $\mathrm{mm}^{3}$, with 10,753 polynuclear neutrophils per $\mathrm{mm}^{3}$ ) and C-reactive protein $75 \mathrm{mg} \cdot \mathrm{L}^{-1}$ (normal range $<5 \mathrm{mg} \cdot \mathrm{L}^{-1}$ ), with no renal failure or hepatic biological abnormalities. Her gamma globulin level was normal $\left(11.7 \mathrm{~g} \cdot \mathrm{L}^{-1}\right)$. Aspergillosis serology and the tuberculin intradermal reaction were negative; sputum examination was sterile, without Mycobacterium or Aspergillus. Chest radiography showed linear opacity of the right upper lobe with bronchiectasis and excavated opacity in the right upper lobe. A computed tomography scan of the chest revealed bronchiectasis with a thickened lining and cystic dilatation of the right upper lobe, as well as diffuse centrolobular micronodules in all lobes of the right lung (fig. 1).

On bronchoscopy the bronchial mucous membrane was normal but had a suppurating secretion. Microbiological evaluation of bronchoscopic samples did not reveal any Mycobacterium or Aspergillus species, but did reveal a Nocardia species identified as Nocardia cyriacigeorgica from 16S rRNA gene sequencing in the French Nocardiosis Observatory (Lyon, France). Antibiotic susceptibility was tested. The isolate was sensitive to amoxicillin-clavulanic acid (minimum inhibitory concentration (MIC): $1.2 \mu \mathrm{g} \cdot \mathrm{mL}^{-1}$ ), cefotaxime (MIC: $0.04 \mu \mathrm{g} \cdot \mathrm{mL}^{-1}$ ), amikacin (MIC: $0.01 \mu \mathrm{g} \cdot \mathrm{mL}^{-1}$ ) and trimethoprim-sulfamethoxazole (MIC: $\left.0.002 \mu \mathrm{g} \cdot \mathrm{mL}^{-1}\right)$.

An intravenous antimicrobial therapy of trimethoprimsulfamethoxazole (cotrimoxazole) and amikacin was administered for 15 days followed by oral only trimethoprimsulfamethoxazole for 6 months. The patient's clinical condition gradually improved over the course of her stay in the hospital. At 4 months after discharge from the hospital, follow-up radiological investigations showed a favourable outcome. Alveolar condensation and centrolobular micronodules were resolved, but cystic bronchiectasis persisted within the fine lining (fig. 1). The clinical, biological and radiological outcomes were still favourable, without a relapse 12 months after the end of antimicrobial therapy.

A pulmonary infection caused by Aspergillus can cause the same radiological abnormalities in patients suffering from COPD as 